\title{
ENHANCED VEGETATION INDEX (EVI) NA ANÁLISE DA DINÂMICA DA VEGETAÇÃO DA RESERVA BIOLÓGICA DE SOORETAMA, ES ${ }^{1}$
}

\author{
André Quintão de Almeida², Gilson Fernandes da Silva³, José Eduardo Macedo Pezzopane ${ }^{3}$ e Carlos
} Alexandre Damasceno Ribeiro ${ }^{4}$

\begin{abstract}
RESUMO - Técnicas de análises de séries temporais são utilizadas para caracterizar o comportamento de fenômenos naturais no domínio do tempo. Neste artigo, segundo a metodologia proposta por Box et al. (1994), 125 observações do Enhanced Vegetation Index (EVI) foram analisadas. Os valores modelados correspondem às variações temporais ocorridas no dossel florestal da reserva biológica de Sooretama, localizada ao Norte do Estado do Espírito Santo, no Município de Linhares. Os resultados indicaram que a metodologia foi adequada. Os resíduos do modelo ajustado são não correlacionados com distribuição normal, média zero e variância $\sigma^{2}$. Com o menor valor do Critério de Informação de Akaike (AIC) -570,51, o modelo ajustado foi o Sazonal Auto-Regressivo Integrado de Médias Móveis $(1,0,1)(1,0,1)_{12}$.
\end{abstract}

Palavras-chave: Sensoriamento remoto, Box e Jenkins.

\section{USE OF ENHANCED VEGETATION INDEX (EVI) IN THE ANALYSIS OF VEGETATION DYNAMICS OF THE SOORETAMA BIOLOGICAL RESERVATION, ES}

\begin{abstract}
Temporal series analysis techniques are used to characterize the behavior of natural phenomenon in time domain. In this paper, 125 Enhanced Vegetation Index (EVI) observations were analyzed according to the methodology proposed by Box et al.(1994). The values modeled correspond to the temporal variations that occurred in the forest canopy of the Sooretama Biological Reserve, in northern Espirito Santo, in the district of Linhares. The results indicated that such methodology was adequate. The residues of the adjusted model are not correlated with normal distribution, zero average and $\sigma^{2}$ variance. At the lowest value of the Akaike Information Criteria (AIC) -570. 51, the model adjusted was the Mobile Average Integrated Self-Regressive Seasonal model $(1,0,1)(1,0,1)_{-12}$.
\end{abstract}

Keywords: Remote sensing, GIS, Box and Jenkins.

\section{INTRODUÇÃO}

Imagens multitemporais de sensoriamento remoto, transformadas em índices de vegetação (VIs), são ferramentas poderosas usadas para monitorar mudanças ocorridas na cobertura vegetal (KARNIELI et al., 2002). O Enhanced Vegetation Index (EVI) é utilizado na avaliação do vigor da vegetação, pois está diretamente relacionado com variações ocorridas na cobertura verde (JUSTICE et al., 1998). Imagens EVI são geradas por meio das bandas individuais na faixa espectral do azul, vermelho e infravermelho do sensor Moderate Resolution Imaging Spectroradiometer (MODIS). Tal índice minimiza os efeitos de resposta do solo e atmosfera, como também apresenta alta resposta a variações fenológicas (JUSTICE et al., 1998). Os dados são formados a partir de diversas observações da área de interesse de um período de 16 dias, sendo então, após o processamento, livres de coberturas de nuvens.

\footnotetext{
${ }^{1}$ Recebido em 01.02.2007 e aceito para publicação em 22.08.2008.

${ }^{2}$ Programa de Pós-Graduação em Engenharia Agrícola da Universidade Federal de Viçosa (UFV). E-mail: <andreqa@ gmail.com>.

${ }^{3}$ Centro de Ciências Agrárias da Universidade Federal do Espírito Santo (UFES), Alegre-ES. E-mail: <gfsilva@cca.ufes.br>

${ }^{4}$ Programa de Pós-Graduação em Produção Vegetal da UFES.
} 
O EVI é obtido pela seguinte equação (JUSTICE et al., 1998)

$\mathrm{EVI}=2,5(I V P-v e r) /(L+I V P+C 1$ ver $-C 2 a z u l), \quad 1$ em que L é fator de ajuste para o solo; C1 e C2 são coeficientes de ajuste para efeito de aerossóis da atmosfera; $a z u l$ é a reflectância da região espectral do azul, veré a reflectância da região espectral do vermelho e IVP é a reflectância da região espectral do infravermelho próximo. Os valores dos coeficientes dos coeficientes são $\mathrm{L}=1, \mathrm{C} 1=6, \mathrm{C} 2=7,5$ e o fator de ganho $\mathrm{G}=2,5$ (JUSTICE et al., 1998).

Em muitos dos estudos com dados de sensoriamento remoto, os que são relacionados com o domínio do tempo têm proporcionado melhores resultados diante dos casos de domínio espacial, radiométrico e espectrais. Ao utilizar séries temporais de imagens de sensoriamento remoto, a fim de analisar o comportamento fenológico da vegetação, várias são as abordagens utilizadas, desde análise textural e análises de componentes principais até a utilização de métricas que descrevem as mudanças sazonais dos índices de vegetação (HUETE et al., 2002).

Considerando o exposto, foi objetivo desta pesquisa aplicar técnicas de sensoriamento remoto e análises de séries temporais para monitorar as variações temporais em um fragmento florestal de Mata Atlântica de Tabuleiros. A hipótese considerada é de que a série do EVI, juntamente com a metodologia estatística empregada (BOX et al., 1994), seja ferramenta adequada para avaliar mudanças na floresta dentro do domínio do tempo.

\section{MATÉRIAL E MÉTODOS}

\section{1. Área de estudo}

A área de floresta estudada está localizada no Município de Sooretama, Norte do Espírito Santo, entre as latitudes $18^{\circ} 54^{\prime}$ e $19^{\circ} 04^{\prime}$ S e longitudes $39^{\circ} 54^{\prime} \mathrm{e}$ $40^{\circ} 15^{\prime} \mathrm{O}$ (Figura 1). Possui vegetação denominada Floresta Ombrófila Densa das Terras Baixas, também chamada de "Mata dos Tabuleiros", dentro da Província Atlântica, com as árvores variando entre 28 e $60 \mathrm{~m}$ de altura. Esse tipo de floresta é formada sobre rochas pré-cambrianas e recobertas por sedimentos inconsolidados de origem continental, depositados durante o Terciário Superior e início do quaternário - o Grupo Barreiras (AMADOR, 1982).

O clima é do tipo tropical quente úmido, com estação chuvosa no verão e seca no inverno. A temperatura média anual é de $23{ }^{\circ} \mathrm{C}$. O relevo nessa região origina feições representadas por uma seqüência de colinas tabulares (interflúvios tabulares), entrecortadas por vales amplos e rasos, podendo-se identificar uma única unidade geomorfológica denominada Tabuleiros Costeiros, que se caracterizam por formas aplainadas, parcialmente conservadas, submetidas a retoque e remanejamentos sucessivos.

\subsection{Pré-processamento das imagens}

As imagens utilizadas são produtos do sensor MODIS relacionados ao estudo da vegetação. O EVI é produzido globalmente com resolução de $250 \mathrm{~m}$ e composições no período de 16 dias. O período analisado foi de abril de 2000 a outubro de 2005, totalizando 127 observações. Esses dados estão disponíveis em Earth Observing System Data Gateway (http:// edcimswww.cr.usgs.gov/pub/imswelcome/).

As imagens, originalmente no formato Hierarchy Data Format (HDF) e na projeção sinusoidal contínua, foram convertidas para o formato Geo-tiff e para a projeção Universal Transversa de Mercator (UTM), através do software MODIS Reprojection Tool. Apresentando um nível de quantificação de 12 bits, fez-se necessária a transformação para o nível de 8 bits. A ferramenta utilizada para conversão de quantificação foi o software ConvGeotiff (ARAI, 2002). Essa transformação é necessária para que o software SPRING possa analisar as imagens, que, após esse processo, foram importadas do referido software.

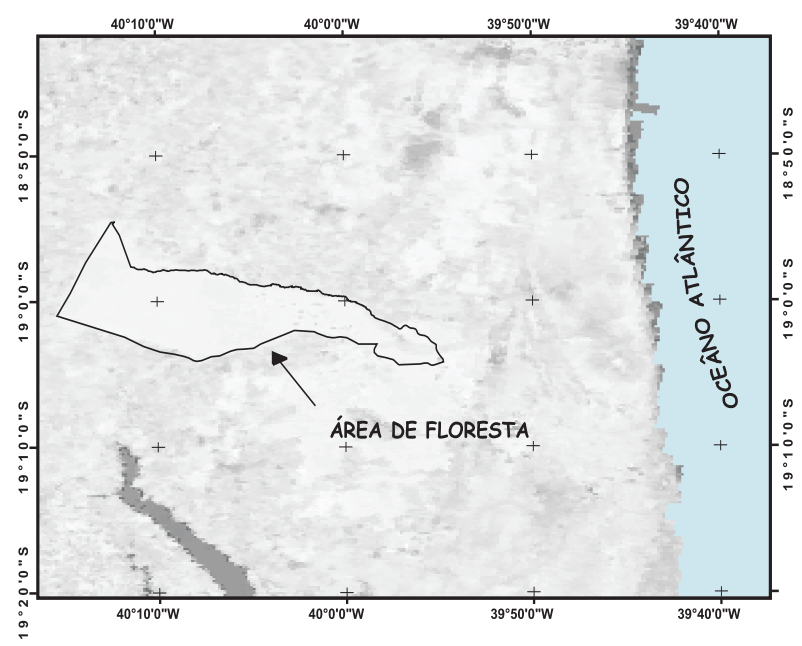

Figura 1 - Imagem MODIS, produto EVI e localização da área de estudo.

Figure 1-MODIS image, EVI product and study area location. 


\subsection{Obtenção dos valores de EVI}

$\mathrm{Na}$ área em estudo, foram gerados pontos amostrais (193) de forma aleatória, a partir dos quais se coletaram os respectivos valores de brilho. Esses pontos repetiramse nas 127 imagens, sendo transformados em valores EVI, através da fórmula 2/256*brilho- 1 . A partir desses 193 valores de EVI, foi gerado um valor médio de EVI para cada imagem. Esses valores médios compuseram a série temporal, a qual passou por um filtro de mediana de 3 , que por sua vez excluiu o primeiro e o último valor da série. Resultando na série temporal final, composta por 125 observações de valores médios de EVI.

\subsection{Modelagem estatística de séries temporais (Box e Jenkins)}

De modo geral, toda série temporal $\left(Y_{t}, t=1: \mathrm{n}\right)$ pode ser decomposta em uma componente de tendência, outra sazonal e uma aleatória, do tipo $Y_{t}=T_{t}+S_{t}+$ $a_{t}$. A tendência $\left(T_{t}\right)$ pode ser entendida como aumento ou diminuição gradual nos valores da série ao longo do período; a série com sazonalidade $\left(S_{t}\right)$ apresenta repetição de comportamento ao longo do tempo e $a_{t}$, a componente aleatória ou erro. Espera-se que $a_{t}$ seja um ruído branco com média zero e variância constante.

$\mathrm{Na}$ prática, geralmente as séries apresentam tendência e, ou, sazonalidade. Na maioria dos casos, ao apresentarem essas componentes, a série é dita não estacionária ${ }^{1}$. A condição de estacionariedade é necessária para garantir a invertibilidade do processo, ou seja, para que os valores projetados possam ser transformados em sua base original.

Segundo a metodologia de Box et al. (1994), dada uma série não-estacionária na média $Z_{t}$, comumente se utiliza o operador diferença $\nabla$ para tornar a série estacionária. Para um inteiro $d \geq 0$, esse operador atua da forma dada na equação (2).

$$
\nabla^{d} Z_{t}=(1-B)^{d} Z_{t}
$$

em que $B$ é o operador de atraso definido por $B^{k} Z_{t}=Z_{t-k}$.

Um processo $Z_{t}$ diz-se um modelo auto-regressivo integrado de médias móveis, abreviado por ARIMA $(p, d, q)$, se satisfaz:

$$
\phi(B) \nabla^{d} Z_{t}=\theta(B) a_{t}
$$

em que:

$\phi(B)=1-\phi_{1} B-\phi_{2} B^{2}-\ldots-\phi_{p} B^{p}$ e $\theta(B)=1-\theta_{1} B-\theta_{2} B^{2}-\ldots-\phi_{q} B^{q}$ são polinômios em $B$ de graus $p$ e $q$, chamados, respectivamente, de polinômios auto-regressivos estacionários e médias móveis invertíveis. Os polinômios $\phi(B)$ e $\theta(B)$ devem ter todas as raízes fora do círculo unitário para cumprir as condições de estacionariedade e invertibilidade, respectivamente, e $a_{t}$ deve ser um ruído branco de média zero e variância constante.

Quando um padrão dinâmico de sazonalidade estocástica está presente na série, ele pode ser modelado com pequena modificação sobre o modelo ARIMA, dado na equação 3 . Essa modificação tem a seguinte forma:

$$
\phi(B) \Phi\left(B^{s}\right) \nabla^{d} \nabla_{s}^{D} Z_{t}=\theta(B) \Theta\left(B^{s}\right) a_{t}
$$

em que:

$$
\begin{aligned}
& \Phi\left(B^{s}\right)=1-\Phi_{1} B^{s}-\ldots-\Phi_{P} B^{s^{P}} \\
& \Theta\left(B^{s}\right)=1-\Theta_{1} B^{s}-\ldots-\Theta_{Q} B^{s^{Q}} \\
& \Delta_{s}^{D}=\left(1-B^{s}\right)^{P}
\end{aligned}
$$

em que o processo $W_{t}=\nabla^{d} \nabla_{s}^{D} Z_{t}$ é estacionário e $d$ e $D$ representam o número de diferenças simples e sazonal feitas sobre $Z_{t}$ com o período sazonal $s$.

A idéia central da técnica Box et al. (1994) é a de que uma série temporal pode ser parcialmente explicada por ela mesma, por suas realizações anteriores (parâmetros auto-regressivos) e pelos próprios erros (parâmetros de médias móveis).

O modelo dado na equação 4 é denominado AutoRegressivo Integrado de Médias Móveis Sazonais, SARIMA $(p, d, q) \times(P, D, Q)$. Não há, em princípio, nenhuma dificuldade adicional na identificação, estimação e verificação de modelos sazonais. Caso seja necessário, pode-se tomar a diferença da série com respeito a $\nabla$ e $\nabla_{s}$, a fim de produzir estacionariedade.

\footnotetext{
${ }^{1} \mathrm{O}$ conceito de estacionariedade significa que "a série se desenvolve no tempo aleatoriamente em torno de uma média constante, refletindo alguma forma de equilíbrio estável”(KASSOUF, 1988).
} 
A partir da obtenção da estacionariedade da série, o próximo passo consiste na identificação do processo gerador da respectiva série temporal. Através da análise da função das autocorrelações regular e parcial ${ }^{2}$, procurase identificar se o processo é auto-regressivo e, ou, de médias móveis, de que ordem é e se apresenta sazonalidade.

Em estudos florestais, os modelos propostos por Box e Jenkins são amplamente utilizados. Silva e Silva(1996) aplicaram o método de Box e Jenkins à série de preço do metro cúbico de carvão vegetal em Minas Gerais, no período de janeiro de 1980 a dezembro de 1992 . Considerando também preços médios do metro cúbico de carvão para o Estado de Minas Gerais, Coelho Junior (2006) considerou o modelo SARIMA, com o objetivo de encontrar os parâmetros que forneçam melhores previsões de preço. Já em Reisen (2006) foi discutida a estimação de processos fracionariamente integrados com componentes sazonais. Portanto, a utilização do modelo SARIMA em estudos ambientais e teóricos tem apresentado resultados consistentes na modelagem e previsão de séries temporais, mostrando ser uma ferramenta útil e motivando novos trabalhos.

\section{RESULTADOS E DISCUSSÃO}

\subsection{Identificação, estimação e adequação do modelo}

Ao analisar a série da Figura 2, percebe-se, com o passar do tempo, comportamento aleatório dos valores médios de EVI. O comportamento dinâmico observado na série de tempo é a resposta fenológica do dossel florestal analisado. Essa resposta varia no tempo em razão do espectro de reflexão da cobertura vegetal incidente nos sensores remotos.

O início e duração das distintas fenofases, como o desenvolvimento, descoloração e senescência das folhas e floração, variam de ano para ano, dependendo das condições climáticas. Entretanto, as fenofases geralmente apresentam comportamento sazonal, ou seja, repetição e, ou, periodicidade anual. Analisando as Figuras 2 e 3, têm-se os picos de EVI, ocorrendo nas mesmas épocas do ano, com máximas $(0,6098)$ no verão e mínimas $(0,4009)$ no inverno, o que caracteriza um comportamento sazonal. A análise estatística das funções de autocorrelações (Figura 4) evidencia a sazonalidade na série.

Ainda interpretando a Figura 2, percebe-se que a pluviosidade, na região em estudo, possui sazonalidade concentrando-se durante o verão. Mudanças sazonais, na disponibilidade dos recursos naturais, atuam de forma potencializadora ou limitante no crescimento da vegetação, alterando a taxa de acumulação de biomassa e a taxa de senescência (LEE et al., 2002).

A pluviosidade, sobretudo, atua de forma marcante sobre a disponibilidade de água no ambiente, regulando processos ecofisiológicos da planta como a fotossíntese e a evapotranspiração; por conseqüência, a assimilação e disponibilidade de fotoassimilados estruturais e de reserva são influenciados. Assim, pode-se observar, na região estudada, a forte relação de dependência entre vigor da vegetação e pluviosidade, em que os valores de EVI se apresentam maiores durante o período chuvoso.

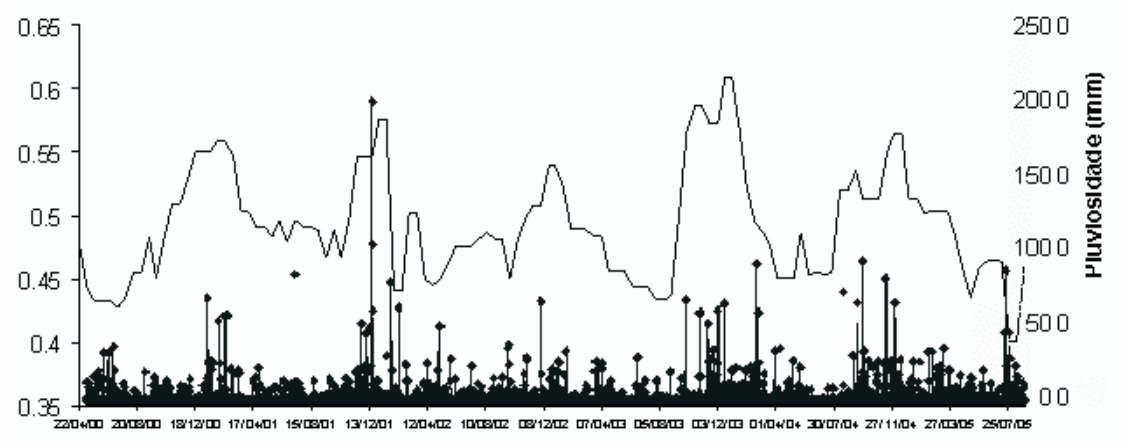

Figura 2 - Séries temporais de valores de EVI e precipitação mensal acumulada entre março de 2000 e setembro de 2005. Figure 2 - Time series of EVI values and accumulated monthly precipitation from March 2000 to September 2005.

${ }^{2}$ Conforme Margarido (1994), a "função de autocorrelação (ACF) indica o processo, enquanto a função de autocorrelação parcial (PACF) mostra a ordem desse processo, no caso de modelos auto-regressivos. No caso de modelos de médias móveis, tem-se o inverso".

R. Árvore, Viçosa-MG, v.32, n.6, p.1099-1107, 2008 


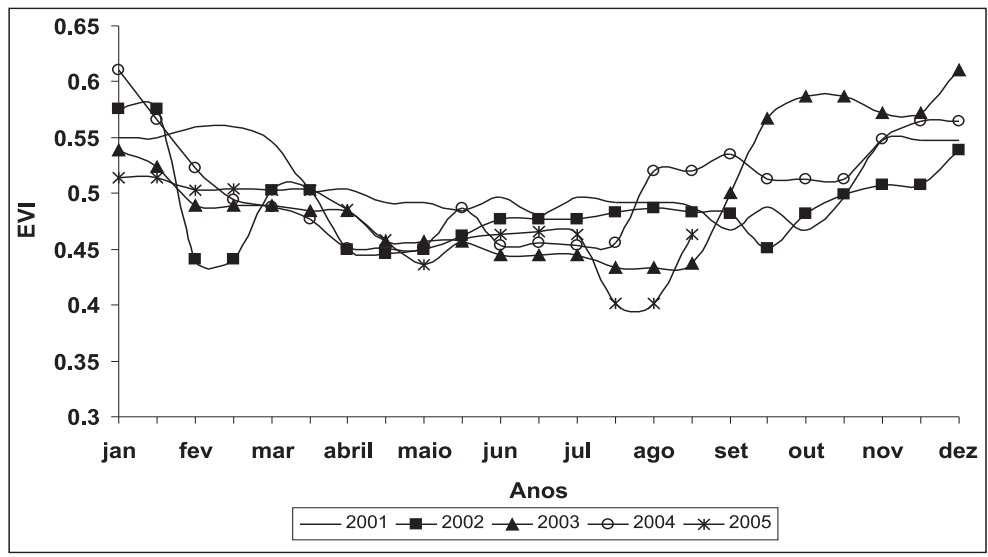

Figura 3 - Valores de EVI dos respectivos anos analisados. Figure 3 - EVI values for the respective years analyzed.
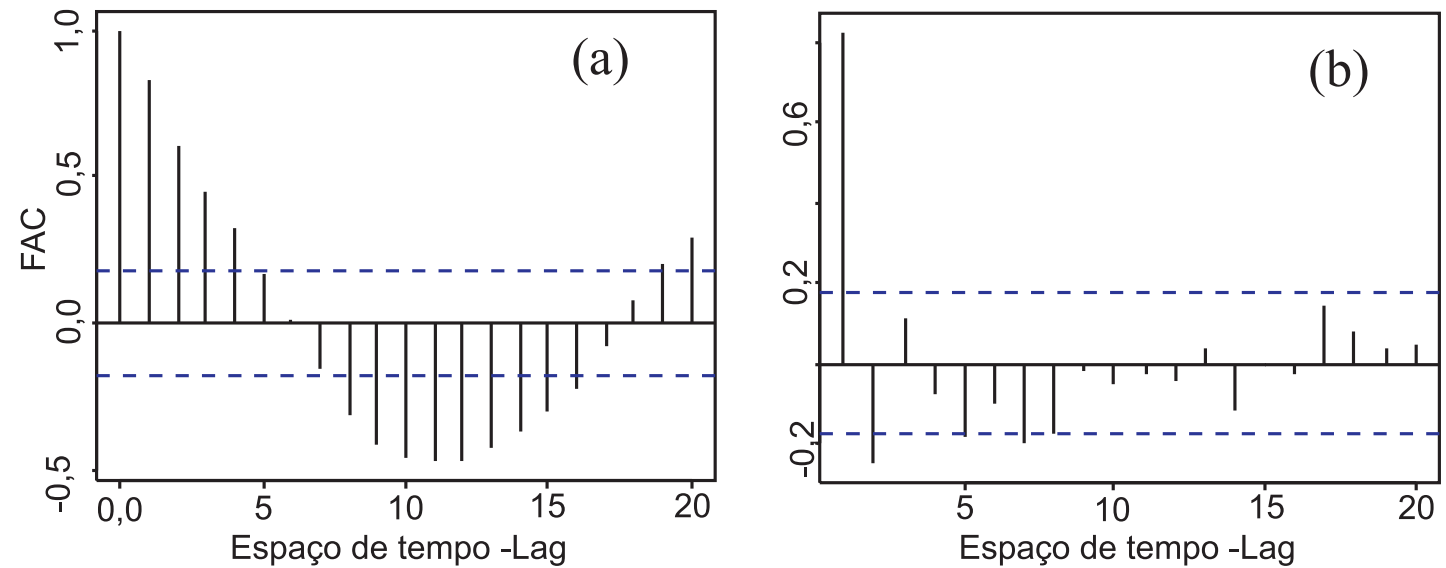

Figura 4 - Funções de autocorrelação (FAC) (a) amostral e autocorrelação parcial (FACP) (b) amostral ajustadas da série de EVI na reserva florestal de Sooretama.

Figure 4-Functions of sampled autocorrelation $(A C F)($ a) and sampled partial autocorrelation (PACP) (b) adjusted from the EVI series in the Sooretama forest reservation.

A FAC (Figura 4a) sugere um modelo do tipo SARIMA, com período de repetição de 12 meses ( $\mathrm{s}=12$ ), que pode ser visualizada pelo resultado dos "lags" 12 significativos, ou seja, fora do intervalo de significância representado pela linha pontilhada. A FACP (Figura 4b) sugere também que o coeficiente auto-regressivo ("p") do modelo seja igual a 1, visto que apresenta defasagem de tamanho 1 significativa.

A estacionariedade da série foi confirmada pelo teste de raiz unitária Dickey-Fuller Aumentado (ADF)
(DICKEY, 1979). A hipótese nula, em que o processo observado possui pelo menos uma raiz unitária, foi rejeitada em nível de 5\% de probabilidade. Não sendo necessária a diferenciação da série, tem-se que o coeficiente diferençável do modelo será zero $(\mathrm{d}=0)$.

Após a simulação de vários modelos, comparação de suas variâncias e respectivos valores de Critério de Informação de Akaike 3 (AIC) (AKAIKE, 1974), obtevese um indício de que o melhor modelo para a série é do tipo SARIMA $(1,0,1)(1,0,1)_{12}$. A Tabela 1 sumariza os modelos confrontados.

\footnotetext{
${ }^{3}$ Pelo AIC, o modelo que possuir a menor estatística será escolhido para ajustar o conjunto de dados.
} 
Tabela 1 - Modelos ajustados, variâncias estimadas e valores da estatística AIC

Table 1 -Adjusted models, dear variances and values of statistics AIC

\begin{tabular}{llllllcc}
\hline Modelo & $p$ & $q$ & $P$ & $D$ & $Q$ & S$^{2}$ estimada & AIC \\
\hline SARIMA & 1 & 1 & 2 & 0 & 1 & 291,27 & $-568,53$ \\
SARIMA & 1 & 1 & 1 & 0 & 2 & 291,27 & $-568,54$ \\
SARIMA & 1 & 1 & 1 & 0 & 0 & 288,84 & $-567,68$ \\
SARIMA & 1 & 1 & 0 & 0 & 1 & 288,18 & $-566,36$ \\
SARIMA & 0 & 1 & 1 & 0 & 1 & 275,9 & $-541,8$ \\
SARIMA & 1 & 0 & 1 & 0 & 1 & 291,25 & $-570,51$ \\
\hline
\end{tabular}

Para verificar se os coeficientes estimados AR1 $=0,6152, \mathrm{MA} 1=0,4024, \mathrm{MA}_{\text {Sazonal }} 1=-0,8534 \mathrm{e} \mathrm{AR}_{\text {Sazonal }} 1$ $=0,6642$ são necessários ao modelo, foi aplicado o teste " $t$ " 4 . Considerando um nível $\alpha=0,05$, tem que $t_{0,025,124}=1,960$. Com isso, os coeficientes são diferentes de zero, isto é, ambos são significativos. Logo, temse que se o modelo SARIMA $(1,0,1)(1,0,1)_{12}$ ajustado para a série EVI será da forma:

$$
(1-0,6152 B)\left(1-B^{12}\right) Z t=(1-0,4024 B)\left(1+0,85342 B^{12}\right)(
$$

$\left(1-0,6642 B^{12}\right)$ at

Para verificar a adequação de um modelo, a teoria estatística faz pressuposições básicas das propriedades dos seus resíduos (WEI, 1990). Para que o modelo ajustado seja adequado, os resíduos devem apresentar distribuição normal com média zero e variância $\sigma^{2}$, além de serem não-correlacionados. No modelo ajustado, essas características foram avaliadas nos erros encontrados e, portanto, consideradas.

O gráfico apresentado na Figura 5 mostra que os resíduos do modelo apresentam uma distribuição de probabilidade normal com média zero e variância $\sigma^{2}$, evidenciando-se, assim, que o modelo ajustado é adequado.

A estatística de Ljung e Box (LJUNG, 1978) pode ser utilizada para testar a hipótese de não autocorrelação dos resíduos. Se o modelo for apropriado, a estatística terá aproximadamente distribuição $\chi^{2}$. Tem-se que o teste de Ljung-Box (Figura 6) retorna uma resposta positiva, ou seja, os altos valores encontrados fazem com que não se rejeite a hipótese de independência dos resíduos.

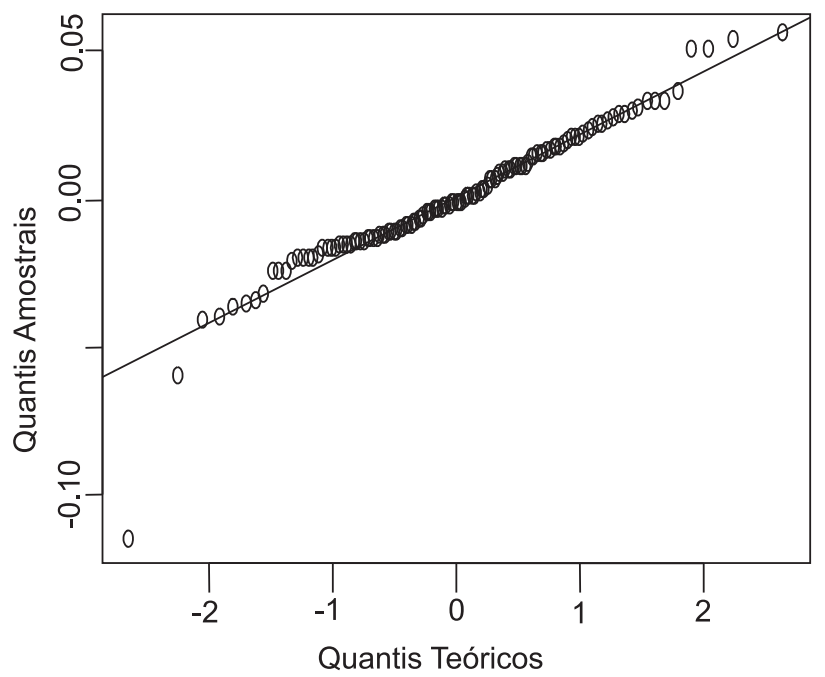

Figura 5 - Q-Q Plot dos resíduos do modelo da equação 8.

Figure $5-Q-Q$ Plot of the residues of the model of the equation 8.

${ }^{4}$ A estatística de teste é $\mathrm{t}$ = coeficiente/erro-padrão do coeficiente. Se a estatística de teste for maior, em módulo, do que o valor t para um nível de confiança $\alpha / 2$ e n - 2 graus de liberdade, então a hipótese nula deverá ser rejeitada.

R. Árvore, Viçosa-MG, v.32, n.6, p.1099-1107, 2008 


\section{Standardized Residuals}

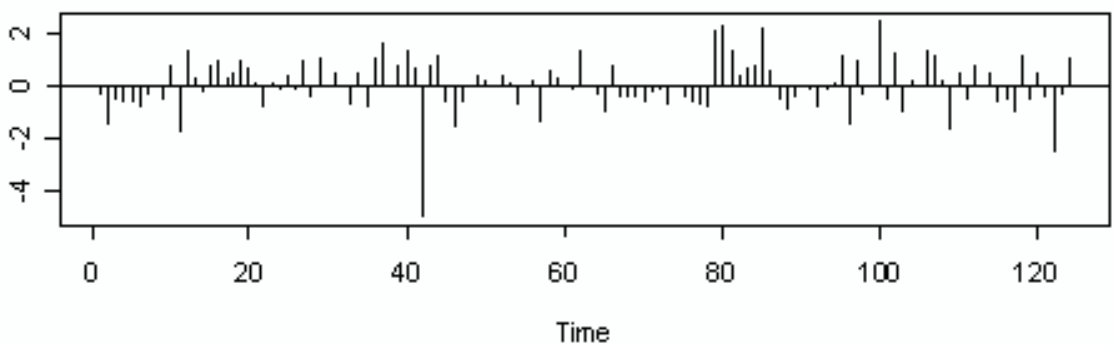

ACF of Residuals

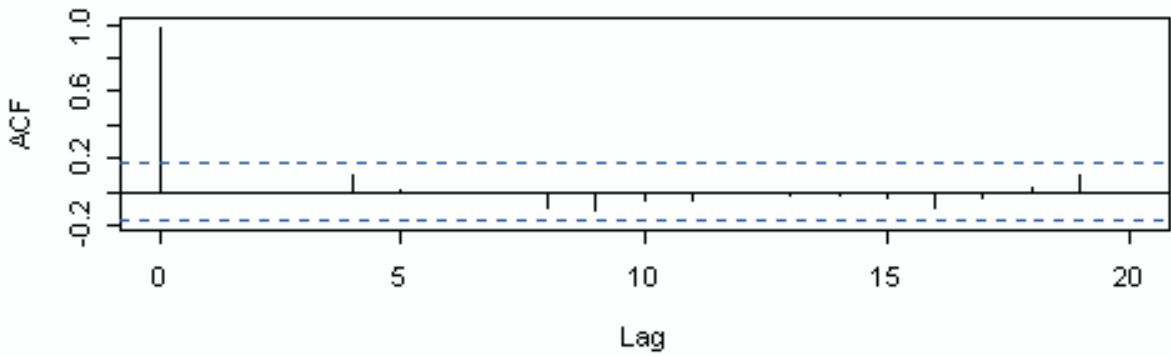

$p$ values for Ljung-Box statistic

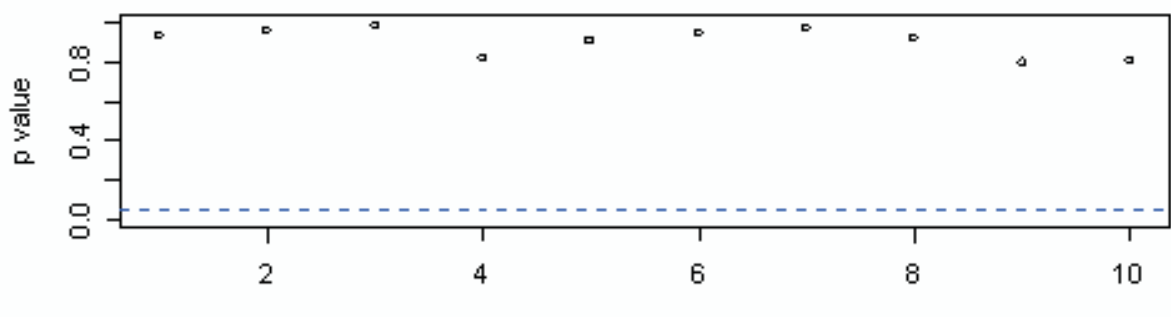

lag

Figura 6 - Diagnóstico do modelo SARIMA $(1,1,2)(0,0,2){ }_{12}$.

Figure 6 - Diagnosis of the SARIMA $(1,1,2)(0,0,2) 12$ model.

Observa-se que todos os gráficos dos resíduos se mostram favoráveis ao modelo ajustado. É possível observar que não há evidências contra a hipótese de ausência de autocorrelação dos resíduos, como também contra a hipótese de normalidade destes.

\section{CONCLUSÕES E RECOMENDAÇÕES}

A partir do trabalho realizado, pôde-se concluir que:

1. Os dados orbitais do sensor MODIS, produtos EVI, representaram as mudanças ocorridas no domínio do tempo no dossel florestal estudado.
2. Os dados do EVI capturaram a dinâmica anual da vegetação, diante dos efeitos do clima.

3. A sazonalidade nos dados, com periodicidade de um ano, foi reconhecida pela metodologia estatística de séries temporais, apresentando periodicidade de um ano.

4. De acordo com os critérios estatísticos, o modelo ajustado foi o SARIMA $(1,0,2)(1,0,1)_{12}$, apresentando o menor valor de AIC (-570,51).

5. A análise dos resíduos do modelo indicou que este é adequado, com uma distribuição de probabilidade normal com média zero e variância $\sigma^{2}$.

R. Árvore, Viçosa-MG, v.32, n.6, p.1099-1107, 2008 
Recomenda-se, em estudos futuros, o uso de séries de tempo com um número superior de observações e, além disso, executar a análise estatística de valores extremos (outliers) na série. Recomenda-se, também, realizar a análise estatística de séries temporais em dados climáticos (precipitação, temperatura etc.) durante o mesmo período de tempo analisado, com o intuito de validar o modelo encontrado e as variações obtidas na série de EVI.

\section{REFERÊNCIAS}

AKAIKE, H. A new look at statistical model identification. IEEE Transactions

Automatic Control, v.19, n.6, p.716-723, 1974.

AMADOR, E. S. Depósitos relacionados à unidade inferior do Grupo Barreiras no Estado do Espírito Santo. In: CONGRESSO BRASILEIRO DE GEOLOGIA, 32., 1982, Salvador, Anais... Salvador: 1982. 1982. v.4. p. 1-61.

ARAI, E. Convgeotiff. São José dos Campos: Instituto Nacional de Pesquisas Espaciais, 2002. (Comunicação pessoal)

BOX, G. E. P.; JENKINS, C. M.; REISEL, G. C. Time series analysis: Forecasting and control. Englewood Cliffs: Prentice Hall, 1994.

DICKEY, D. A.; FULLER, W. A. Distributions of the estimators for autoregressive time series with a unit root. Journal of the American Statistical Association, v.75, n. 366, p.427-431, 1979.

CAMPBELLA, E. P. et al. Statistical forecasting of soil dryness index in the southwest of Western Austrália. Forest Ecology and Management, v.183, 1/3, p.147-157, 2003.

HUETE, A. R. et al. Overview of the radiometric and biophysical performance of the MODIS vegetation indices. Remote Sensing of Environment, v.83, Special Issue. p.195-213, 2002.

COELHO JUNIOR, L. M. et al. Análise longitudinal dos preços do carvão vegetal, no estado de minas gerais. Revista Arvore, v.30, n.3, p.429-438, 2006.

R. Árvore, Viçosa-MG, v.32, n.6, p.1099-1107, 2008
JUSTICE, C. O. et al. The Moderate Resolution Imaging Spectroradiometer (MODIS): land remote sensing for global change research. IEEE Transactions on Geoscience and Remote Sensing, v.36, n.4, p.1228-1249, 1998.

KARNIELI, A. et al. Temporal dynamics of soil and vegetation responses in a semi-arid environment. International Journal of Remote Sensing, v.23, n.19, p.4073-4087, 2002.

KASSOUF, A. L. Previsão de preços na pecuária de corte do estado de São Paulo. São Paulo. 1988. 102p. Dissertação (Mestrado em Economia Aplicada) - Programa de Pós-Graduação em Economia. ESALQ/USP, São Paulo, 1988.

LEE, R.; YU, F.; PRICE, K. P. Evaluating vegetation phonological patterns in Inner Mongolia using NDVI time-series analysis. International Journal of Remote Sensing, v.23, n.12, p.2505-2512, 2002.

LJUNG, G. M.; BOX, G. E. P. On a measure of lack of fit in time series models. Biometrika, v.65, n.2, p.297-303, 1978.

MARGARIDO, M. A. Transmissão de preços internacionais de suco de laranja para preços ao nível de produtor de laranja no Estado de São Paulo. 1994. 96p.Dissertação (Mestrado em Economia de Empresas) - Programa de PósGraduação em Economia. São Paulo: FGV/ EAESP, 1994.

MASELLI, F. Monitoring forest conditions in a protected Mediterranean coastal area by the analysis of ultiyear NDVI data. Remote Sensing of Environment, v.89, n.4, p.423-433, 2004.

REISEN, V. A., RODRIGUES, A. L., PALMA, W. Estimation of seasonal fractionally integrated processes, Computational Statistics \& Data Analysis, v.50, n.2, p.568-582, 2006.

REED, B. C. J. J. et al. In: WORKSHOP CLIMATIC-VEGETATION INTERACTION. Procedings... Maryland: NASA/Goddard space flight center, 1986. p.110-112. 
ROUSE Jr., J.W. et al. Monitoring the vernal advancement and retrogradation (green wave effect) of Natural vegetation. Maryland: NASA/ GSFC, 1974. 371p. (Type III Final Report, 1974)

RUNNING, S. W. et al. A remote sensing based vegetation classification logic for global land cover análisis. Remote Sensing

Environment, v.51, n.1, p.39-48, 1995.
SILVA, M. L.; SILVA, J. M. A. Análise do comportamento temporal dos preços do carvão vegetal: aplicação do modelo e a avaliação da metodologia "Box and Jenkins". Revista Arvore, v.20, n.1, p.57-67, 1996.

WEI, W. W. S. Time series analysis: univariate and multivariate methods. Redwood City: Addison-Wesley, 1990. 478p. 